\title{
Boundary value problems on part of a level-n Sierpinski gasket
}

\section{Xuliang Li $i^{*}$}

"Correspondence: xulli8997@163.com

Department of Mathematical Sciences, Tsinghua University, Beijing 100084, China

\section{Springer}

\begin{abstract}
We study the boundary value problems for the Laplacian on a sequence of domains constructed by cutting level-n Sierpinski gaskets properly. Under proper assumptions on these domains, we manage to give an explicit Poisson integral formula to obtain a series of solutions subject to the boundary data. In particular, it is proved that there exists a unique solution continuous on the closure of the domain for a given sequence of convergent boundary values.
\end{abstract}

MSC: 28A80; 35J25

Keywords: boundary value problems; level-n Sierpinski gasket; harmonic functions; postcritically finite; fractal Laplacian

\section{Introduction}

The study of boundary value problems on the domains of Sierpinski gasket (SG) was initiated by [1]. Since then, two natural choices have been considered, namely the upper part of SG cut by a horizontal line (cf. [1, 2]) and half Sierpinski gasket constructed by cutting SG with a vertical line in the middle (cf. [3]). For more related works see, for example, [4-8]. This work is strongly motivated by [3].

In this work, we will introduce a new class of domains on level-n Sierpinski gasket and prove the exact form of the solution to the boundary value problems on these domains. Note that these domains are new examples of non-p.c.f. (postcritically finite) type fractals (can also be viewed as fractafold in $[9,10]$ ) where harmonic functions can be well defined.

We follow $[11,12]$ by recalling that the fractal $K$ is the invariant set for a finite iterated function systems (IFS) of contractive similarities in the Euclidean space $\mathbb{R}^{2}$. We denote the mappings $\left\{F_{i}\right\}_{i=0, \ldots . N-1}$ for some positive integer $N$. Then $K$ is the unique nonempty compact set satisfying

$$
K=\bigcup_{i=0}^{N-1} F_{i}(K)
$$

For $m \geq 1$, we define the space of words of length $m$ by

$$
W_{m}^{N}=\{0,1,2, \ldots, N-1\}^{m}=\left\{w_{1} w_{2} \ldots w_{m}: w_{i} \in\{0,1,2, \ldots, N-1\}\right\} .
$$

(c) The Author(s) 2017. This article is distributed under the terms of the Creative Commons Attribution 4.0 International License (http://creativecommons.org/licenses/by/4.0/), which permits unrestricted use, distribution, and reproduction in any medium, provided you give appropriate credit to the original author(s) and the source, provide a link to the Creative Commons license, and indicate if changes were made. 
$w \in W_{m}^{N}$ is called a word of length $m$ with symbols $\{0,1,2, \ldots, N-1\}$. We also set $W_{*}^{N}=$ $\bigcup_{m \geq 0} W_{m}^{N}$ and denote the length of $w \in W_{*}^{N}$ by $|w|$.

Recall that $K$ is called postcritically finite (p.c.f.) if $K$ is connected and there exists a finite set $V_{0} \subseteq K$ called the boundary such that

$$
F_{w} K \cap F_{w^{\prime}} K \subseteq F_{w} V_{0} \cap F_{w^{\prime}} V_{0} \quad \text { for } w \neq w^{\prime} \text { with }|w|=\left|w^{\prime}\right|,
$$

with the intersection disjoint from $V_{0}$. Set $V_{0}=\left\{q_{0}, q_{1}, \ldots, q_{N_{0}}\right\}$ for $N_{0}<N$. We require that each boundary point is the fixed point of one of the mappings $\left\{F_{i}\right\}$ and that

$$
F_{i}\left(q_{i}\right)=q_{i} \quad \text { for } 0 \leq i \leq N_{0}
$$

The standard SG is the unique nonempty compact set $K$ satisfying (1.1) with the boundary set $V_{0}=\left\{q_{0}, q_{1}, q_{2}\right\}$, where the contractive mappings $\left\{F_{i}\right\}_{i=0,1,2}$ are given by

$$
F_{i}(x)=\frac{1}{2}\left(x-q_{i-1}\right)+q_{i-1} .
$$

Similarly, the level-3 Sierpinski gasket $\mathrm{SG}_{3}$ is the unique nonempty compact set $K$ satisfying (1.1) with the boundary set $V_{0}=\left\{q_{0}, q_{1}, q_{2}\right\}$, where $\left\{F_{i}\right\}_{i=0 . ., 5}$ are given by

$$
F_{i}(x)=\frac{1}{3}\left(x-q_{i}\right)+q_{i}
$$

Here $q_{3}=\frac{q_{1}+q_{2}}{2}, q_{4}=\frac{q_{0}+q_{2}}{2}, q_{5}=\frac{q_{0}+q_{1}}{2}$. See Figure 2 for an illustration.

As above, we can define level-n Sierpinski gasket in a similar way.

Inspired by [3] we will construct a new class of domains in the following statement.

\subsection{Description of the general domains}

Let $K=\mathrm{SG}_{n}$ and $\tilde{K}=\frac{1}{n} K$, that is, shrinking $K n$ times. Denote by $\widetilde{E}$ the compact triangular domain with boundary set $\left\{q_{0}, q_{1}, q_{2}\right\}$ which is constructed by gluing finite copies of $\tilde{K}$ at boundary (see Example 1.3 below). Assume that the compact triangular domain $\widetilde{E}_{1}$ with boundary $\left\{q_{0}, q_{1}^{\prime}, q_{2}^{\prime}\right\}$ (as a part of $\widetilde{E}$ ) satisfies $\widetilde{E}_{1}=F_{0}(\widetilde{E})$, where $F_{0}(x):=q_{0}+\frac{x-q_{0}}{L}$ for some constant $L$ (see Figure 1(a)). Pick some point $\hat{q}$ such that the contractive map

$$
\widehat{F}(x):=\widehat{q}+\frac{x-\widehat{q}}{L}
$$

Figure 1 General domain.

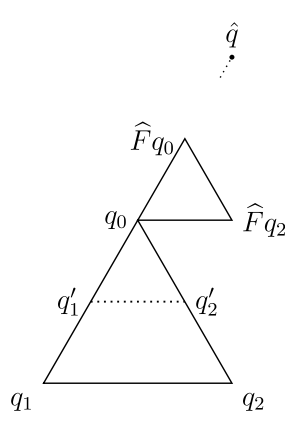

(a) Iteration pattern

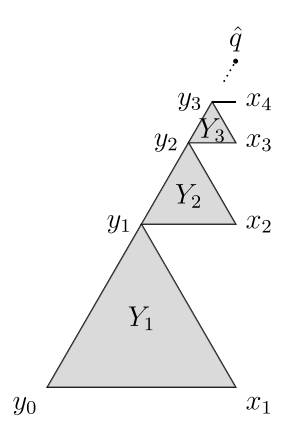

(b) Resulted domain 
satisfies $\widehat{F}\left(q_{1}\right)=q_{0}$. Set $E=\widetilde{E} \backslash\left\{q_{1}, q_{2}\right\}, y_{0}=q_{1}, y_{m}=\widehat{F}^{m}\left(q_{1}\right), x_{m}=\widehat{F}^{m-1}\left(q_{2}\right), Y_{m}:=\widehat{F}^{m-1}(E)$ for integer $m>0$ (see Figure 1(b)). Let $X=\bigcup_{m=1}^{\infty}\left\{x_{m}\right\}$. Set

$$
\partial Y_{m}=\left\{y_{m-1}, y_{m}, x_{m}\right\}, \quad \bar{Y}_{m}=Y_{m} \cup \partial Y_{m} .
$$

For each $\bar{Y}_{m}$ define mapping $F_{m}$ as

$$
F_{m}(z)=y_{m}+\frac{z-y_{m}}{L} .
$$

Then by assumption $\bar{Y}_{m}$ is self-similar with respect to $F_{m}$. Define

$$
\Omega:=\bigcup_{m=0}^{\infty} Y_{m}, \quad \bar{\Omega}:=\bigcup_{m=0}^{\infty} \bar{Y}_{m} .
$$

We say that the set $\Omega$ is a DCPB (domain of countable-point boundary) with boundary $\partial \Omega:=X \cup y_{0} \cup \hat{q}$ for $K=\mathrm{SG}_{n}$.

Remark 1.1 In application, we need the constant $L=n^{k}$ with $k>0$ to ensure that $\widetilde{E}$ is self-similar with respect to the map $F_{0}$, and thus $F_{m}\left(\bar{Y}_{m}\right)$ is exactly a copy of $\bar{Y}_{m+1}$. This property is useful in constructing harmonic functions on these domains in a sequel. The description of those domains will be justified by the examples below.

\subsection{Examples}

Example 1.2 For $K=\mathrm{SG}_{3}$, let $\widetilde{E}=F_{1}(K)$ be the compact triangular domain with boundary set $\left\{q_{1}, p_{1}, p_{6}\right\}$ (see Figure 2). Let $E=\widetilde{E} /\left\{q_{1}, p_{1}\right\}$, set $y_{0}=q_{1}, y_{m}=F_{5}\left(q_{1}\right), x_{m}=F_{5}^{m-1}\left(p_{1}\right)$ and $Y_{m}=F_{5}^{m-1}(E)$ for all positive integers $m$, where $F_{5}$ is as defined in (1.4). Set $\widehat{F}=F_{5}, \hat{q}=q_{5}$. Then $\Omega$ (green part) can be well established as in (1.6).

Example 1.3 For $K=\mathrm{SG}_{3}, \widetilde{E}=F_{1}(K) \cup F_{3}(K) \cup F_{5}(K)$ is the compact triangular domain with boundary set $\left\{q_{1}, p_{2}, p_{5}\right\}$ (see Figure 2). Let $E=\widetilde{E} /\left\{q_{1}, p_{2}\right\}$, set $y_{0}=q_{1}, y_{m}=F_{0}^{m}\left(q_{1}\right)$, $x_{m}=F_{0}^{m-1}\left(p_{2}\right)$ and $Y_{m}=F_{0}^{m-1}(E)$ for all positive integers $m$. Let $X=\bigcup_{m=1}^{\infty}\left\{x_{m}\right\}$. Setting $\widehat{F}=F_{0}, \hat{q}=q_{0}$, we obtain $\Omega$ (gray part) as a DCPB by (1.6).

Example 1.4 For $K=\mathrm{SG}_{2}, \widetilde{E}=F_{1}(K)$ is the compact triangular domain with boundary points $\left\{q_{1}, p_{2}, p_{0}\right\}$ (see Figure 3). Let $q_{4}=\frac{2 p_{2}+q_{0}}{3}$ and define the contractive mapping

$$
F_{4}(x)=\frac{x-q_{4}}{4}+q_{4}
$$

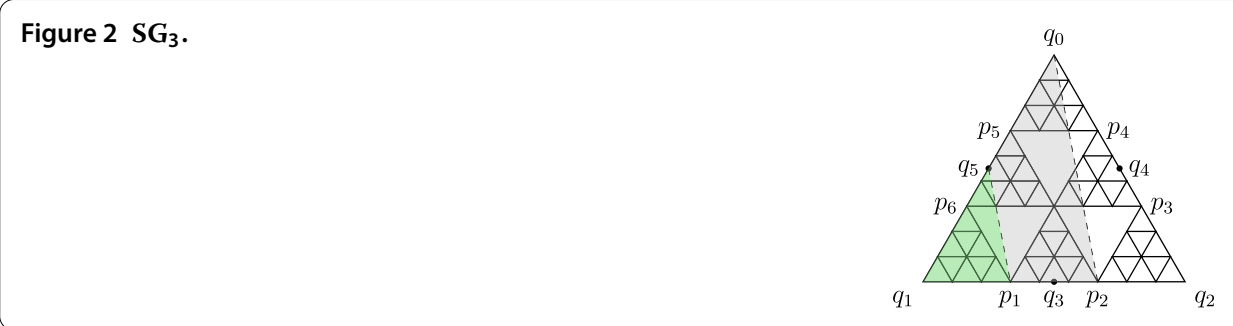


Figure 3 Domain in $\mathbf{S G}_{2}$.

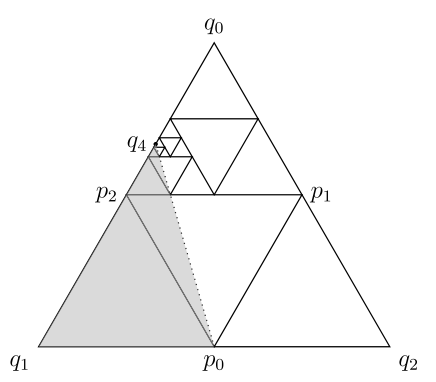

Let $E=\widetilde{E} /\left\{q_{1}, p_{0}\right\}$, set $y_{0}=q_{1}, y_{m}=F_{4}^{m}\left(q_{1}\right), x_{m}=F_{4}^{m-1}\left(p_{0}\right)$ and $Y_{m}=F_{4}^{m-1}(E)$ for all $m>0$. Let $X=\bigcup_{m=1}^{\infty}\left\{x_{m}\right\}$. Set $\widehat{F}=F_{4}, \hat{q}=q_{4}$. Now we can define the desired domain $\Omega$ by (1.6). Note that $F_{4}$ is not one of the contractive mappings for standard SG.

In the following section, we construct a solution to the boundary value problem using harmonic extension algorithm. Denote by $C(U)$ the space of all continuous functions on some set $U$. We will see that the space of $C(\Omega)$-solutions to the boundary value problem is one-dimensional, but in general, the solution blows up at $\hat{q}$. We show that if the boundary data on $X$ converges, there exists a unique $C(\bar{\Omega})$-solution.

\section{Main results}

The Laplacian on the standard SG was first constructed as a generator of a stochastic process by Goldstein [13] and Kusuoka [14]. Kigami [15, 16] developed an analytical version of the Laplacian for SG, and then generalized it to any p.c.f. self-similar set (see [11], Definition 3.7.1, p.108).

We now study the boundary value problems on $\Omega$ as a DCPB defined in Section 1.1:

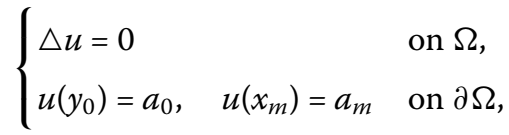

where $\triangle$ denotes the Kigami's Laplacian for $K=\mathrm{SG}_{n}$ with respect to the standard selfsimilar measure, $u: \bar{\Omega} \rightarrow \mathbb{R}$ is the unknown, and $\left\{a_{m}\right\}_{m=0}^{\infty}$ is the boundary data. Note that the Laplacian $\Delta$ here is well defined for all cells $Y_{m}$, hence the whole $\Omega$ by recalling that every cell $\bar{Y}_{m}$ of $\Omega$ can be viewed as a part of $K=\mathrm{SG}_{n}$ or gluing several copies of it.

Harmonic extension algorithm is the simplest tool for constructing harmonic functions subject to boundary value problems on $\mathrm{SG}_{n}$. In fact, we can apply this algorithm infinitely many times and obtain a function harmonic on $\mathrm{SG}_{n}$.

Using this, we will give an explicit solution to (BVP) based on the following assumption.

Assumption 0 Let $\Omega$ be a DCPB for $K=\mathrm{SG}_{n}$. For each cell $Y_{m}$ with boundary set $\partial \bar{Y}_{m}=$ $\left\{y_{m-1}, y_{m}, x_{m}\right\}$, if some function $u$ is harmonic on $Y_{m}$ and satisfies that

$$
u\left(y_{m}\right)=c_{1}, \quad u\left(y_{m-1}\right)=c_{2}, \quad u\left(x_{m}\right)=c_{3}
$$




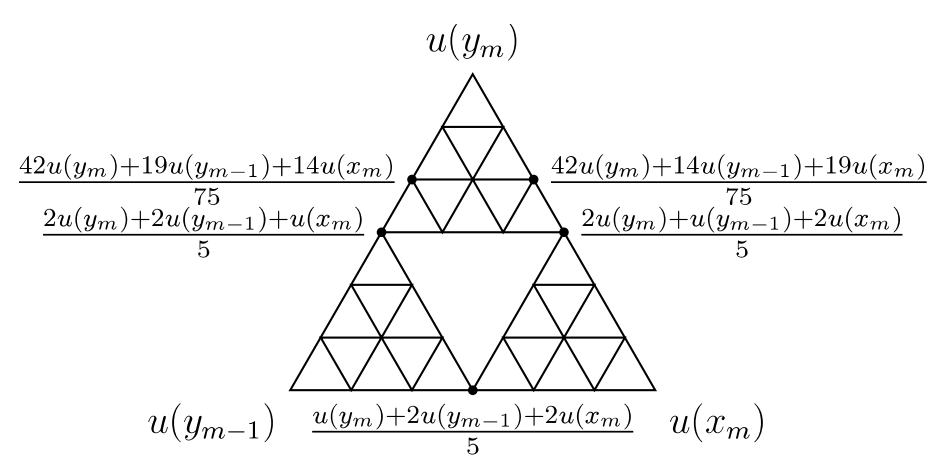

Figure 4 Values for Assumption 0 in Example 1.3.

for some real constants $c_{1}, c_{2}, c_{3}$, then

$$
\left[\begin{array}{c}
u\left(y_{m}\right) \\
u\left(y_{m-1}^{\prime}\right) \\
u\left(x_{m}^{\prime}\right)
\end{array}\right]=M_{0}\left[\begin{array}{c}
u\left(y_{m}\right) \\
u\left(y_{m-1}\right) \\
u\left(x_{m}\right)
\end{array}\right]=M_{0}\left[\begin{array}{c}
c_{1} \\
c_{2} \\
c_{3}
\end{array}\right], \quad M_{0}=\left[\begin{array}{ccc}
1 & 0 & 0 \\
\theta_{1} & \theta_{2} & \theta_{3} \\
\theta_{1} & \theta_{3} & \theta_{2}
\end{array}\right]
$$

for some positive constants $\theta_{1}, \theta_{2}, \theta_{3}$ satisfying that

$$
\theta_{1}+\theta_{2}+\theta_{3}=1
$$

where $y_{m-1}^{\prime}=F_{m}\left(y_{m-1}\right), x_{m}^{\prime}=F_{m}\left(x_{m}\right)$ with $F_{m}$ given by (1.5).

Note that this assumption can be easily verified by harmonic extension algorithm. In Example 1.3, we have (see Figure 4)

$$
M_{0}=\left[\begin{array}{ccc}
1 & 0 & 0 \\
\frac{42}{75} & \frac{19}{75} & \frac{14}{75} \\
\frac{42}{75} & \frac{14}{75} & \frac{19}{75}
\end{array}\right]
$$

We set, for Assumption 0,

$$
\begin{array}{ll}
\Theta_{0}=\theta_{2}+\theta_{3}, & \Theta_{1}=2\left(2-\theta_{1}\right), \\
T_{+}=\frac{\Theta_{1}+K}{2}, & T_{-}=\frac{\Theta_{1}-K}{2}, \quad K=\sqrt{\Theta_{1}^{2}-4 \Theta_{0} .}
\end{array}
$$

Theorem 2.1 For every choice of the convergent boundary data $\left\{a_{m}\right\}$ for some $\Omega$ as a DCPB (defined in Section 1.1) satisfying Assumption 0, there exists a one-dimensional space of $C(\Omega)$ solutions to the (BVP). For each real constant $\lambda$, there exists a unique solution to the (BVP) $u_{\lambda}$ such that $u_{\lambda}\left(y_{1}\right)=\lambda$ and that $u_{\lambda}\left(x_{m}\right)=a_{m}$ for $m \geq 1$. Furthermore, for $m \geq 2$

$$
u_{\lambda}\left(y_{m}\right)=K^{-1}\left\{T_{+}^{m} \phi_{m}^{+}(\lambda)-T_{-}^{m} \phi_{m}^{-}(\lambda)\right\},
$$


where

$$
\begin{aligned}
& \phi_{m}^{+}(\lambda)=\lambda-T_{+}^{-1} \Theta_{0}\left(a_{0}+a_{1}\right)-\left(\Theta_{0}+T_{+}\right) \sum_{k=2}^{m} T_{+}^{-k} a_{k}, \\
& \phi_{m}^{-}(\lambda)=\lambda-T_{-}^{-1} \Theta_{0}\left(a_{0}+a_{1}\right)-\left(\Theta_{0}+T_{-}\right) \sum_{k=2}^{m} T_{-}^{-k} a_{k} .
\end{aligned}
$$

Proof For fixed $m \geq 2$, let $u$ be a continuous piecewise harmonic function for (BVP). In view of Assumption 0, it is easy to adapt the argument for [3], proof of Lemma 2.1, to obtain that $\Delta u\left(y_{m}\right)=0$ holds if and only if

$$
u\left(y_{m}\right)=\Theta_{1} u\left(y_{m-1}\right)-\Theta_{0} u\left(y_{m-2}\right)-a_{m}-\Theta_{0} a_{m-1} .
$$

The rest is trivial algebra as in [3], proof of Theorem 2.2.

The theorem below can be obtained by following the argument in [3], proof of Theorem 2.4, Corollary 2.5. We include a brief proof for the readers' convenience.

Theorem 2.2 If $a_{m} \rightarrow A$ as $m \rightarrow \infty$ for some constant $A$, there exists a unique solution to (BVP) $u \in C(\bar{\Omega})$ which satisfies that

$$
u\left(y_{1}\right)=T_{+}^{-1} \Theta_{0}\left(a_{0}+a_{1}\right)+\left(\Theta_{0}+T_{+}\right) \sum_{k=2}^{\infty} T_{+}^{-k} a_{k},
$$

and for $m \geq 2$

$$
\begin{aligned}
u\left(y_{m}\right)= & K^{-1}\left(\Theta_{0}+T_{+}\right)\left\{\sum_{k=1}^{\infty} T_{+}^{-k} a_{m+k}-T_{-}^{m} \sum_{k=2}^{\infty} T_{+}^{-k} a_{k}\right\} \\
& +T_{-}^{m}\left\{a_{0}+a_{1}+K^{-1}\left(\Theta_{0}+T_{-}\right) \sum_{k=2}^{m} T_{-}^{-k} a_{k}\right\} .
\end{aligned}
$$

Proof We first prove the theorem for the case $A=0$.

Substituting (2.6) into (2.4) yields (2.7).

By using the triangle inequality, we have

$$
\begin{aligned}
\left|u\left(y_{m}\right)\right| \leq & K^{-1}\left(\Theta_{0}+T_{+}\right)\left\{\sum_{k=1}^{\infty} T_{+}^{-k}\left|a_{m+k}\right|-T_{-}^{m} \sum_{k=2}^{\infty} T_{+}^{-k}\left|a_{k}\right|\right\} \\
& +T_{-}^{m}\left\{\left|a_{0}\right|+\left|a_{1}\right|+K^{-1}\left(\Theta_{0}+T_{-}\right) \sum_{k=2}^{m} T_{-}^{-k}\left|a_{k}\right|\right\} .
\end{aligned}
$$

From this and $a_{m} \rightarrow 0$ we can easily see that $u\left(y_{m}\right) \rightarrow 0$. Thus, by the definition of BVP,

$$
\lim _{m \rightarrow \infty} u\left(x_{m}\right)=\lim _{m \rightarrow \infty} u\left(y_{m}\right)=0 .
$$

It follows by [3], Lemma 2.3, that $u \in C(\bar{\Omega})$. Since harmonic functions that are continuous to the boundary satisfy the maximum principle [17], we obtain the uniqueness by the standard argument for linear differential equations satisfying the maximum principle. 
For the case $A \neq 0$, we consider the modified BVP:

$$
\begin{cases}\triangle u=0 & \text { on } \Omega \\ u\left(y_{0}\right)=a_{0}-A, \quad u\left(x_{m}\right)=a_{m}-A & \text { on } \partial \Omega\end{cases}
$$

Noting that $a_{m}-A \rightarrow 0$, the rest of the proof can be done by using the result from the last part of the proof and the maximum principle.

Remark 2.3 The results in [3], Section 2, reduce to a special case of our theorems above with parameters $\theta_{0}=2 / 5, \theta_{1}=2 / 5, \theta_{2}=1 / 5$. Indeed, [3] proved many more results on half SG. Many of them are highly dependent on the fact that we can obtain SG from half SG by reflection, and thus the top point enjoys many more nice properties than our domains. We will touch on that elsewhere.

\section{Competing interests}

The author declares that he has no competing interests.

\section{Author's contributions}

The author read and approved the final manuscript.

\section{Acknowledgements}

The author is indebted to Professor Robert S. Strichartz for inviting them to work on this topic. The author is also grateful to Professor Jiaxin Hu for helpful comments on a draft version of the present work. This work was supported by the National Natural Science Foundation of China (11371217).

\section{Publisher's Note}

Springer Nature remains neutral with regard to jurisdictional claims in published maps and institutional affiliations.

Received: 18 January 2017 Accepted: 28 March 2017 Published online: 05 April 2017

\section{References}

1. Owen, J, Strichartz, RS: Boundary value problems for harmonic functions on a domain in the Sierpinski gasket. Indiana Univ. Math. J. 61(1), 319-335 (2012)

2. Guo, Z, Kogan, R, Qiu, H, Strichartz, RS: Boundary value problems for a family of domains in the Sierpinski gasket. III. J. Math. 58(2), 497-519(2014)

3. Li, W, Strichartz, RS: Boundary value problems on a half Sierpinski gasket. J. Fractal Geom. 1(1), 1-43 (2014)

4. Bonanno, G, Bisci, GM, Radulescu, V: Existence results for gradient-type systems on the Sierpiński gasket. Chin. Ann. Math., Ser. B 34(2), 941-953 (2013)

5. Bonanno, G, Molica Bisci, G, Radulescu, V: Infinitely many solutions for a class of nonlinear elliptic problems on fractals. C. R. Math. Acad. Sci. Paris 350(3-4), 187-191 (2012)

6. Bonanno, G, Molica Bisci, G, Radulescu, V: Variational analysis for a nonlinear elliptic problem on the Sierpiński gasket. ESAIM Control Optim. Calc. Var. 18(4), 941-953 (2012)

7. Ferrara, M, Molica Bisci, G, Repovs, D: Existence results for nonlinear elliptic problems on fractal domains. Adv. Nonlinear Anal. 5(1), 75-84 (2016)

8. Molica Bisci, G, Radulescu, V: A characterization for elliptic problems on fractal sets. Proc. Am. Math. Soc. 143(7), 2959-2968 (2015)

9. Strichartz, RS: Fractafolds based on the Sierpiński gasket and their spectra. Trans. Am. Math. Soc. 355(10), 4019-4043 (2003)

10. Strichartz, RS: Fractals in the large. Can. J. Math. 50(3), 638-657 (1998)

11. Kigami, J: Analysis on Fractals. Cambridge Tracts in Mathematics, vol. 143. Cambridge University Press, Cambridge (2001)

12. Strichartz, RS: Differential Equations on Fractals: A Tutorial. Princeton University Press, Princeton (2006)

13. Goldstein, S: Random walks and diffusions on fractals. In: Percolation Theory and Ergodic Theory of Infinite Particle Systems (Minneapolis, Minn., 1984-1985). IMA Vol. Math. Appl., vol. 8, pp. 121-129. Springer, New York (1987)

14. Kusuoka, S: Dirichlet forms on fractals and products of random matrices. Publ. Res. Inst. Math. Sci. 25(4), 659-680 (1989)

15. Kigami, J: A harmonic calculus on the Sierpiński spaces. Jpn. J. Appl. Math. 6(2), 259-290 (1989)

16. Kigami, J: Harmonic calculus on p.c.f. self-similar sets. Trans. Am. Math. Soc. 335(2), 721-755 (1993)

17. Strichartz, RS: Some properties of Laplacians on fractals. J. Funct. Anal. 164(2), 181-208 (1999) 